Abstract P5-S4.05 Table 1 Comparison of characteristics of infectious syphilis cases $(N=155)$

\begin{tabular}{|c|c|c|c|c|}
\hline \multirow[b]{2}{*}{ Factor } & \multicolumn{4}{|l|}{ Number of cases } \\
\hline & $\begin{array}{l}\text { Outreach cases, } \\
n=10(\%)\end{array}$ & $\begin{array}{l}\text { Routine testing } \\
\text { cases } n=145(\%)\end{array}$ & $\begin{array}{l}\text { Total, } \\
N=155(\%)\end{array}$ & p Value \\
\hline \multicolumn{5}{|l|}{ Gender } \\
\hline Female & $8(80.0)$ & $68(46.9)$ & $76(49.0$ & 0.05 \\
\hline Male & $2(20.0)$ & $77(53.1)$ & $79(51.0)$ & \\
\hline \multicolumn{5}{|l|}{ Ethnicity } \\
\hline Aboriginal & $7(70.0)$ & $58(40.0)$ & 65 (41.9) & 0.10 \\
\hline Non-aboriginal & $3(30.0)$ & $87(60.0)$ & $90(58.1)$ & \\
\hline Median age (IQR) & $32.5(21.25-37.5)$ & $32(24-43)$ & $32(24-42)$ & 0.71 \\
\hline \multicolumn{5}{|l|}{ Risk behaviour } \\
\hline Heterosexual & $10(100.0)$ & $107(83.6)$ & $117(84.8)$ & 0.16 \\
\hline Injection drug use & $2(20.0)$ & $11(7.6)$ & $13(8.4)$ & 0.20 \\
\hline Sex with an IDU & 0 & $8(5.5)$ & $8(5.2)$ & 1.00 \\
\hline \multicolumn{5}{|l|}{ Sex worker } \\
\hline Female & $6(75.0)$ & $16(23.5)$ & $22(28.9)$ & 0.006 \\
\hline Male & 0 & $1(1.3)$ & $1(1.3)$ & 1.00 \\
\hline $\begin{array}{l}\text { Sex with a sex } \\
\text { worker (males only) }\end{array}$ & $2(100.0)$ & $22(28.6)$ & $24(30.4)$ & 0.09 \\
\hline
\end{tabular}

Results A total of 931 testing visits were completed with 877 individuals. Three-quarters of individuals reported a history of substance use $(77.1 \%$; $n=676)$ with more than one-quarter of individuals involved in transactional sex, either as a sex worker $(n=204)$ or contact with a sex worker $(n=110)$. Over half of the individuals $(51.3 \% ; n=450)$ were Aboriginal. Ten newly diagnosed infectious syphilis cases were found. The proportion of cases detected among female sex workers was $2.9 \%$ ( 6 cases out of 209 testing visits) and was $2.0 \%$ (2 cases out of 98 testing visits) among male sexual contacts of sex workers. Female cases were more likely to be a sex worker when tested through outreach (75\%) than by routine testing sites $(23.5 \%, \mathrm{p}=0.006)$ see Abstract P5-S4.05 table 1 .

Conclusions By offering testing at community agencies with financial compensation to individuals, a higher rate of cases were found among sex workers than through routine testing methods. By treating cases among individuals linked to high transmission activity, we aim to reduce the transmission of infectious syphilis.

\section{P5-S4.06 STICKING TO IT: THE EFFECT OF MAXIMALLY ASSISTED THERAPY ON ANTIRETROVIRAL TREATMENT ADHERENCE AMONG A COHORT OF UNSTABLY HOUSED PEOPLE LIVING WITH HIV IN BC, CANADA}

doi:10.1136/sextrans-2011-050108.547

${ }^{1} \mathrm{~S}$ Parashar, ${ }^{1} \mathrm{~A}$ Palmer, ${ }^{1} \mathrm{~N}$ O'Brien, ${ }^{1} \mathrm{~K}$ Chan, ${ }^{1} \mathrm{~A}$ Shen, ${ }^{2} \mathrm{~S}$ Coulter, ${ }^{1,3} \mathrm{~J}$ Montaner, ${ }^{4} \mathrm{R}$ Hogg. ${ }^{1} B C$ Centre for Excellence in HIVIAIDS, Vancouver, Canada; ${ }^{2}$ Vancouver Coastal Health Authority, Canada; ${ }^{3} \mathrm{UBC}$, Department of Medicine, Canada; ${ }^{4} \mathrm{SFU}$, Faculty of Health Sciences, Canada

Background Housing is a known determinant of health behaviour, including adherence to antiretroviral therapy. Within the Longitudinal Investigations into Supportive and Ancillary Health Services (LISA) cohort, unstable housing is inversely associated with adherence. The Maximally Assisted Therapy (MAT) program uses a multidisciplinary approach to support people living with HIV/AIDS (PHA) who have a history of addictions, mental health disorders and homelessness. We investigated the efficacy of support services, including the MAT program, in improving adherence for unstably housed PHA.

Methods The LISA cohort is a cross-sectional study of individuals on antiretroviral therapy in British Columbia. Interviewer-administered surveys collect information regarding housing, drug use, utilisation of health services and other clinically relevant socio-demographic factors. Clinical variables, such as CD4 count and viral load, were obtained through longitudinal linkages with the Drug Treatment Program (DTP) at the BC Centre for Excellence in HIV/AIDS. Logistic regression was used to determine factors associated with adherence ( $\geq 95 \%$ vs $<95 \%$ ) among unstably housed LISA participants $(\mathrm{n}=212)$.

Results Between 2007 and 2010 approximately 1000 participants were interviewed. This analysis is based on 644 interviews, of which the DTP reports optimal adherence [ $\geq 95 \% 12$ month refill] for 367 (57\%) individuals. Median age was 46 and 475 (73.7\%) were male. We found that unstably housed participants attending the MAT program were 4.76 times more likely to be $\geq 95 \%$ adherent [95\% CI 1.72 to 13.13$]$ than those who did not. Other factors associated with optimal adherence included recent incarceration (Adjusted OR $[\mathrm{AOR}]=0.20$ [95\% CI 0.05 to 0.80$]$ ) and not currently using illicit drugs ( $\mathrm{AOR}=0.40[95 \% \mathrm{CI} 0.16$ to 0.99$]$ ).

Conclusion The MAT program provides a model for other urban centers dealing with concurrent and interrelated adherence barriers: high-risk drug use, mental health disorders and homelessness. In the absence of sustainable housing solutions, programs such as MAT are crucial to achieving optimal treatment adherence in this population.

\section{P5-S4.07 SMS REMINDERS INCREASE RE-TESTING FOR REPEAT CHLAMYDIAL INFECTION IN HETEROSEXUALS AT A SEXUAL HEALTH CLINIC}

doi:10.1136/sextrans-2011-050108.548

${ }^{1} \mathrm{R}$ Guy, ${ }^{1} \mathrm{H}$ Wand, ${ }^{2} \mathrm{P}$ Read, ${ }^{2} \mathrm{~A}$ Kenigsberg, ${ }^{2} \mathrm{~V}$ Knight, ${ }^{2,3} \mathrm{~A}$ McNulty. ${ }^{1}$ National Centre in HIV Epidemiology and Clinical Research, Sydney, Australia; ${ }^{2}$ Sydney Sexual Health Centre, Sydney Hospital, Sydney, Australia; ${ }^{3}$ School of Public Health and Community Medicine, University of NSW, Sydney, Australia

Background Repeat infection with Chlamydia trachomatis following treatment is common. If left untreated it can lead to onward transmission and in females it increases the risk of pelvic inflammatory disease by fourfold. In late 2008, Sydney Sexual Health Centre implemented a reminder system using short message service (SMS) to improve re-testing rates following treatment of chlamydial infection. Clinicians were advised to set up SMS reminders to be sent out at 3 months after the visit.

Methods We evaluated the impact of this system in its first year of operation using a controlled observation design. Using logistic regression we assessed the association between a SMS reminder and re-testing within 1-4 months of chlamydia infection in Jan-August 2009 among all heterosexuals. The comparison group was heterosexuals diagnosed with chlamydia who received a brief recommendation to re-test in the same time period.

Results There were 230 heterosexuals diagnosed with chlamydial infection in the study period; 128 females (median age $=24$ years) and 112 males (median age $=26$ years). Of these, $47 \%$ were sent a SMS reminder (SMS group), and $53 \%$ received a brief recommendation (comparison group). The re-testing rate within 1-4 months of chlamydial infection was $58 \%$ in the SMS group, compared to $42 \%$ in the comparison group, $\mathrm{OR}=1.9,95 \% \mathrm{CI} 1.1$ to $3.2, \mathrm{p}=0=002$. In females, the chlamydia re-testing rate within 1-4 months of chlamydial infection was $57 \%$ in the SMS group, compared to $40 \%$ in the comparison group, $\mathrm{OR}=2.0,95 \% \mathrm{CI} 1.0$ to $4.0, \mathrm{p}=0.05$. In males, the chlamydia re-testing rate within 1-4 months of chlamydial infection was $59 \%$ in the SMS group, compared to $45 \%$ in the comparison group, $\mathrm{OR}=1.7,95 \%$ CI 0.8 to $3.8, \mathrm{p}=0.165$.

Conclusion This low cost system substantially increased re-testing rates after chlamydia infection in heterosexuals at a large sexual health clinic. The clinic now plans to implement electronic prompts to remind clinicians to offer SMS reminders to all patients diagnosed with chlamydia. 